\section{PTH-124 HEPATIC ANGIOSARCOMA IN SOUTH GLAMORGAN: A RARE TUMOUR STILL APPEARING 40 YEARS AFTER OCCUPATIONAL EXPOSURE}

doi:10.1136/gut.2011.239301.525

R Stacey,1,* M Hassell, ${ }^{1} \mathrm{~J}$ T Green ${ }^{1}{ }^{1}$ Department of Gastroenterology, University Hospital Llandough, Llandough, UK; ${ }^{2}$ HM Coroner, Cardiff \& The Vale of Glamorgan Coroner's Court, Cardiff, UK

Introduction Hepatic angiosarcoma is a rare condition with a poor prognosis and an incidence ranging from 0.14 to 0.25 per million ${ }^{1}$ however it occurs more commonly in certain geographical areas. These centre on petrochemical processing plants which were involved in the polymerisation of vinyl chloride to make PVC. Workers acting as 'polycleaners' in the vats were particularly susceptible. Changes were made to the processing schedule and cleaning regimes in 1974, however the authors are continuing to see cases some four decades after occupational exposure. Hepatic angiosarcoma is an industrial disease therefore all deaths have to be reported to the coroner.

Our hospital is close to a petrochemical processing plant in Barry, South Glamorgan and following a recent admission of a man with associated liver failure the authors reviewed the number of cases of hepatic angiosarcoma during the last 15 years in the Cardiff and Vale of Glamorgan area to assess whether or not this was an isolated case.

Methods The authors identified all cases of hepatic angiosarcoma in the Cardiff and Vale of Glamorgan area in the last 15 years via a computer database search of records with the assistance of the coroner.

Results Seven cases of angiosarcoma were identified as outlined in the table 1 below, six of whom had definite exposure to vinyl chloride via the petrochemicals industry, the other was uncertain.

Conclusion Cases of hepatic angiosarcoma related to the petrochemical industry are still occurring and it is important that Gastroenterologists are aware of the continuing presentation of new cases despite changes in safety protocols at petrochemical plants over 35 years ago. This delayed presentation suggests a long latent period between exposure and diagnosis. Occupational history is key to the diagnosis along with a

Table 1 PTH-124

\begin{tabular}{llllll}
\hline Age & Sex & Date of death & Cause of death on death certificate & Exposure & Postmortem verdict \\
\hline 79 & Male & March 1996 & $\begin{array}{l}\text { Fractured cervical spine, but } \\
\text { haemangiosarcoma of liver in part } 2\end{array}$ & Unknown & Accidental death \\
66 & Male & November 2000 & $\begin{array}{l}\text { Hepatic angiosarcoma } \\
\text { Hepatic angiosarcoma }\end{array}$ & $\begin{array}{l}\text { Process supervisor } \\
\text { Chemicals processor }\end{array}$ & $\begin{array}{l}\text { Signed up as a natural cause after PM } \\
\text { Industrial disease }\end{array}$ \\
81 & Male & May 2003 & Angiosarcoma of liver & $\begin{array}{l}\text { Exposed to vinyl chloride } \\
\text { monomer }\end{array}$ & Industrial disease \\
61 & Male & July 2003 & Hepatic angiosarcoma & Exposed to vinyl chloride & Industrial disease \\
59 & Male & May 2004 & Ruptured hepatic angiosarcoma & Chemicals cleaner & Industrial disease \\
77 & Male & October 2009 & Angiosarcoma of liver & Chemicals cleaner & Industrial disease \\
81 & Male & August 2010 & & &
\end{tabular}




\section{BSG abstracts}

typical radiological appearance as biopsy has a high incidence of significant haemorrhage. ${ }^{2}$

Competing interests None.

Keywords hepatic angiosarcoma.

\section{REFERENCES}

1. Molina E, Hernandez A. Clinical manifestations of primary hepatic angiosarcoma. Dig Dis Sci 2003;48:677-82.

2. White PG, Adams H, Smith PM. The computed tomographic appearances of angiosarcoma of the liver. Clin Radiol 1993;48:321-5. 